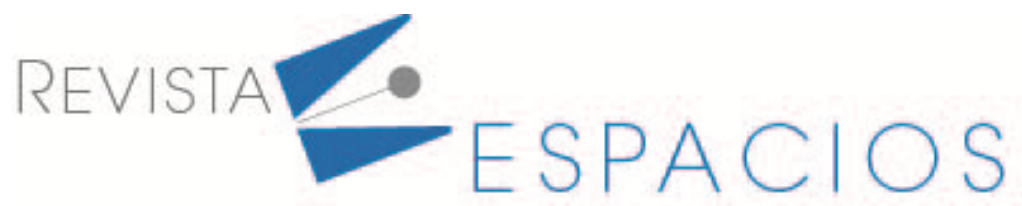

\title{
Los programas de apoyo para el financiamiento del sector agrícola mexicano, caso Sistema Producto Arroz en Morelos (1994-2018)
}

\section{Support programs for financing the Mexican agricultural sector, case of the Rice Product System in Morelos (1994-2018)}

\author{
FERNANDEZ, Alejandra ${ }^{1}$ \\ REYES, Alejandro ${ }^{2}$ \\ ALVAREZ, Fernando ${ }^{3}$
}

\begin{abstract}
Resumen
Durante los últimos veinte años, el sistema agroalimentario mundial ha sufrido grandes transformaciones y la regulación del Estado no ha tenido los resultados esperados. El objetivo del presente estudio es determinar la efectividad de los programas de financiamiento gubernamental. Se realizó un análisis cualitativo, transversal, explorativo y descriptivo del sistema productor arrocero de Morelos, México; concluyendo que las políticas públicas no han tenido la amplitud, el alcance y la profundidad que requieren los productores del estado.

Palabras clave: programas, financiamiento y productores
\end{abstract}

\begin{abstract}
During the last twenty years, the world agri-food system has undergone major transformations and the state regulation has not had the expected results. The objective of this study is to determine the effectiveness of government financing programs. A qualitative, cross, explorative and descriptive analysis was carried out, analyzing the case of the rice farmers of Morelos, Mexico, concluding that public policies have not had the breadth, scope and depth required by state producers

Key words: programs, financing and producers
\end{abstract}

\section{Introducción}

En las últimas dos décadas, las relaciones económicas internacionales han sufrido una gran cantidad de transformaciones, esto ha dado origen a un sistema mundial agroalimentario que se vio obligado a integrar las estructuras agrícolas y agroalimentarias nacionales. Reig (2004), sugirió un análisis que partía de considerar tres aspectos básicos que caracterizaban esta mundialización del sector agroalimentario: su posición en la estructura económica de los países, en la cadena agroalimentaria y la regulación del estado en su estructura y funcionamiento.

\footnotetext{
${ }^{1}$ Doctora en Administración (Negocios Internacionales) por la Universidad Internacional y Profesora de Tiempo Completo de la Universidad Politécnica del Estado de Morelos (UPEMOR)

2 Doctor en Administración (Negocios Internacionales) y Profesor de Tiempo Completo de la Universidad Politécnica del Estado de Morelos (UPEMOR).

${ }^{3}$ Maestro en Gestión Pública Aplicada por el Instituto Tecnológico y de Estudios Superiores de Monterrey (ITESM); Profesor por Asignatura de la Universidad Politécnica del Estado de Morelos (UPEMOR). Email: falvarezganem gmail.com
} 
Las características de las cadenas agroalimentarias (atomicidad, tamaño de las empresas, localización forzosa, riesgos naturales), las obligan a establecer relaciones particulares y profundamente diferentes con cada uno de los agentes involucrados. Sin embargo, se observan patrones de comportamiento representados por demandas oligopsónicas o monopsónicas, cuando industrializan sus productos y oligopólica cuando tecnifican sus procesos y sus proveedores de insumos o bienes de capital son unos cuantos grupos o del estado. Lo anterior, aunque en principio signifique incrementos en la productividad y cambios técnicos, representa importantes fallas de mercado que impiden el intercambio favorable y la poca capacidad de retener las mejoras tecnológicas que se generen. El último aspecto es la regulación por parte del estado. La importancia del medio rural es indiscutible, así como el papel que representa en la producción de alimentos y, por lo tanto, el gobierno lo ha considerado como una de sus mayores prioridades. La relevancia de la seguridad alimentaria es clara, así como su carácter estratégico para cualquier país.

De acuerdo al Banco Mundial, los retos actuales del sector, han señalado la necesidad de crear políticas públicas que permitan que la productividad agrícola pueda abastecer el constante crecimiento de la demanda de alimentos por parte de la población. La inversión que se requiere realizar, también deberá considerar las nuevas tecnologías más intensivas en producción, así como las variedades derivadas de avances en la biotecnología. De acuerdo al Banco Mundial, si los países en vías de desarrollo no realizan inversiones en gran escala en la agricultura y en la creación y difusión del conocimiento, la producción no podrá mantener el ritmo de la demanda y aumentaría la dependencia de la importación de los alimentos que tienen actualmente.

Los programas de apoyo estudiados partieron del gobierno de Miguel de la Madrid Hurtado, en un momento de crisis y endeudamiento, en el que el país adoptaba el modelo neoliberal. Posteriormente, Carlos Salinas de Gortari, creaba políticas públicas para apoyar la apertura comercial a través del tratado de libre comercio con Canadá y Estados Unidos (TLCAN), considerando programas creados desde 1962 como CONASUPO, para cerrar con la gestión de Ernesto Zedillo y Vicente Fox Quezada, con la nueva etapa del programa OPORTUNIDADES.

El análisis se realizó mediante una investigación de campo que se aplicó a los representantes más importantes del Sistema Producto Arroz en Morelos, considerando la amplitud, el alcance y la profundidad de los programas de apoyo

\section{Metodología}

El análisis consideró las variables de amplitud, alcance y profundidad de las políticas públicas.

La amplitud comprende la diversidad de programas que ofrecen apoyo financiero, como los diferentes tipos de crédito de habilitación o avío, refaccionario, simple, hipotecario, prendario o quirografario, entre otros. En lo que respecta a los subsidios, se refierne a los diferentes tipos de apoyos en dinero que otorga el Gobierno Federal a la Banca de Desarrollo (BDSR). La siguiente variable es el alcance y se refiere a la extensión en términos de cantidad, es decir, número de campesinos atendidos, monto del subsidio, superficie financiada, entre otros. En esta investigación, la amplitud se medirá con el número de productores beneficiados, la superficie de hectáreas que cultivan, el número de programas de apoyo que han utilizado, la variedad de destinos que tienen los apoyos (más de diez), entre otros. El último factor fue la profundidad, que es el valor social que se otorga a un cliente o usuario. Se puede calcular a través de las características de los beneficiados: género (las mujeres están consideradas como un grupo vulnerable); grupo étnico (prefiriendo las minorías); zonas (urbana o rural, la segunda debe ser la más favorecida). En este caso se consideraron todos los aspectos antes mencionados en el momento de realizar las preguntas del perfil socioeconómico.

El diseño del estudio requirió evaluar dos componentes estratégicos, el tamaño de la población y la naturaleza de la investigación. Para la definición de la población meta, se utilizó un método no probabilístico debido a que 
se pudo contar con la opinión de líderes arroceros, como el C. Jesús Solís Alvarado, quien ha sido representante del Sistema Producto y del Consejo Regulador del Estado; además, es presidente de la Unión de Productores de arroz del estado de Morelos AC y de la Asociación de arroceros de la región sur del Estado de Morelos USPR (que representan a 600 campesinos, productores y comercializadores del arroz por más de 20 años).

La investigación es un estudio de exploratorio, debido a que no se encuentran documentadas las características del financiamiento del arroz en Morelos. También es descriptiva y la metodología utilizada es de tipo mixto (cuantitativa y cualitativa).

\section{Resultados}

Programas de apoyo a partir de la adopción del modelo neoliberal en México. En México, a principios de 1980, el gobierno del presidente electo, Miguel de la Madrid Hurtado, se enfrentaba a una crisis económica y a otros desafíos importantes. El país había dejado de crecer, registraba el mayor endeudamiento de su historia, teniendo que pagar intereses por un monto mayor al $20 \%$ de los ingresos en divisas derivadas de las exportaciones de bienes y servicios. Por otro lado, se adoptó un modelo neoliberal, por lo que era necesario implementar las medidas necesarias para realizar las reformas estructurales correspondientes. Lo anterior fue realizado exitosamente, ya que actualmente, se considera un sexenio clave para instaurar el estado neoliberal, debido a que sentó las bases necesarias para los cambios posteriores.

La crisis económica que experimentaba el país, provocó que fuera necesario desviar los recursos hacia la atención de los aspectos macroeconómicos derivados de la crisis, evitando atender a otros aspectos como el combate a la pobreza y el desarrollo. El primer ajuste se realizó entre 1983 y 1984, pero no se pudo lograr un gran avance debido, entre otras cosas, a que no se contaba con apoyo financiero del exterior. Para 1986 el país crecía a un $1 \%$ anual, pero su población lo hacía al doble (2\%) y la fuerza laboral a un $3 \%$ anual, por lo que fue imposible evitar que las tasas de desempleo aumentaran significativamente.

El siguiente sexenio (Carlos Salinas de Gortari), se caracterizó por fortalecer las bases para que el modelo funcionara plenamente, logrando la firma del Tratado de Libre Comercio con América del Norte (TLCAN). En lo que respecta a las problemáticas sociales, retoma el estandarte de la solidaridad y aprovecha la experiencia de los programas de la Coordinación General del Plan Nacional de Zonas Deprimidas y Grupos Marginados (COPLAMAR) y el Programa de Inversiones Públicas para el Desarrollo Rural (PIDER).

La COPLAMAR tiene su origen en los años setenta, contemplaba que, a los campesinos, por encontrarse fuera del desarrollo urbano, se les considerara como habitantes de zonas marginadas; y tenía el objetivo de "articular acciones que permitan que las zonas rurales marginadas cuenten con elementos materiales y de organización suficiente para lograr una participación más equitativa de la riqueza nacional". También pretendía que los grupos en condiciones de desventaja alcanzaran una situación de mayor equilibrio. (Cordera \& Lomelí, 2005). COPLAMAR buscaba establecer alianzas con otras dependencias, para crear sinergias con otros sectores pertenecientes a la administración pública. PIDER, por su parte, fue creado en 1973, y pretendía dar respuesta a las siguientes preguntas:

- ¿Cómo puede el gobierno de un país en desarrollo iniciar un proceso de desarrollo rural que se mantenga por sí solo? (Miller, 1985)

- ¿Cómo puede lograrse que las inversiones en el desarrollo rural tengan efectos multiplicadores? (Miller, 1985)

- ¿Cómo puede garantizarse que los beneficios de un programa de desarrollo lleguen realmente a la población que se desea atender sin que se queden en manos de los intermediarios o de los caciques locales? (Miller, 1985)

Se consideraba un programa con un gran potencial para impulsar los procesos de desarrollo, sin embargo, se implementó realizando una gran cantidad de obras y proyectos independientes, que no fueron desarrollados de 
acuerdo a la planeación participativa contemplada inicialmente. En consecuencia, el programa tuvo severas limitaciones culturales y financieras que no pudo superar. Tampoco pudo ser legítimo políticamente, debido a que las alianzas y consensos entre comunidades, municipios, estados y federación, no pudieron llevarse a cabo. (Herrera, 2009)

Otro programa importante fue el del Sistema Alimentario Mexicano (SAM), que tuvo una amplia incidencia en el medio rural, su objetivo era atender la producción, demanda y abasto de alimentos, es decir, enfocó sus esfuerzos en el apoyo a todos los elementos que componen la cadena agroalimentaria. Estuvo vigente de 1980 a 1982, fue un período breve debido a que estaba financiada por recursos petroleros y el sector entró en crisis, por lo que fue necesario realizar un ajuste presupuestal y finalizó el programa.

En 1983 surgieron dos programas, el Programa Nacional Alimentario (PRONAL) y el Programa Nacional de Desarrollo Rural Integral (PRONADRI). El objetivo del PRONAL era reemplazar al SAM, pero tenía menos recursos y menor importancia política. Sus objetivos eran claros, sus estrategias no. No contempló el otorgamiento de subsidios. El PRONADI buscaba ser orientador de las dependencias de la Administración Pública a nivel federal, estatal y municipal partiendo de la participación activa y organizada de las comunidades rurales. También consideraba apoyar a los pequeños productores con sistemas de riego. Ambos programas desaparecieron como resultado de las nuevas políticas derivadas de las reformas estructurales neoliberales.

En 1994, Carlos Salinas de Gortari, sentaba las bases para la consolidación del modelo neoliberal en el país y mejoraba la imagen y percepción de su partido. En lo que respecta a la relación Estado-Sociedad Rural se estaba dando una redefinición, a través de acciones trascendentes como la firma del TLCAN. Para lograrlo, era necesario crear condiciones políticas y económicas y diseñar una nueva forma de organización para las instituciones que pudieran responder a los desafíos que el TLCAN significaba para el medio rural. Por lo tanto, se crearon programas de apoyo, principalmente dirigidos a la comercialización y la atención a los problemas derivados del cambio del art. 27 de la Constitución. Cabe mencionar que la reforma de dicho artículo dio origen a la nueva Ley Agraria, que tenía tres propósitos principales: finalizar el reparto agrario; brindar plena libertar a los ejidos y los habitantes de las comunidades, para que tomaran decisiones autónomas y determinar el orden de la propiedad rural, delimitando la superficie de cada ejido y comunidad, de las tierras de uso común y de los solares de las áreas de asentamiento. (Zorrilla Ornelas, 2003)

Los principales programas sociales del nuevo estado neoliberal surgieron desde el gobierno de Miguel de la Madrid Hurtado, caracterizados por la optimización de recursos para enfrentar la crisis, enfocándose en la atención de la macroeconomía en detrimento de la atención a la pobreza y al desarrollo. Siguieron en orden cronológico: PRONASOL, PROGRESA y OPORTUNIDADES. El Programa Nacional de Solidaridad (PRONASOL), sugería la necesidad de atender situaciones específicas de creación de infraestructura urbana básica.

Los programas de apoyo al campo que se crearon para atender estos desafíos, fueron el Programa de Apoyo Directo al Campo (PROCAMPO), el de Apoyos y Servicios a la Comercialización Agrícola (ASERCA), el Programa Nacional de Modernización del Campo, Programa Nacional Agropecuario y de Desarrollo Rural, Alianza para el campo (APC) y el Programa Especial para la Seguridad Alimentaria (PESA).

El Programa de Apoyo Directo al Campo, surgió en 1993 y funciona hasta la fecha. El objetivo de su creación era coadyuvar a la inserción del país a la dinámica de la globalización. Se consideró el más importante del sexenio de Salinas de Gortari y su vigencia inicial fue de 15 años. El programa representó la libertad comercial, capitalización y competitividad, debido a que permitió la canalización de recursos, por medio de subsidios, a pequeños productores rurales o campesinos. Es decir, permitió la pulverización de recursos al campo, favoreciendo a sectores marginados. Los subsidios eran otorgados de acuerdo al número de hectáreas, sin importar el tipo de cultivo, para poder garantizar que no se distorsionaran los mercados, como sucedió con los programas Sistema 
Alimentario Mexicano (SAM) o el de la Compañía Nacional de Subsistencias Populares (CONASUPO). Esta última surgió en 1962, integró a un gran sistema de abasto que garantizó y reguló los productos de la canasta básica, especialmente el maíz. Durante más de 35 años fue el instrumento gubernamental de abasto más importante del país. Las políticas neoliberales dieron fin a este programa en 1999, cuando fue substituida por LICONSA y DICONSA, de la Secretaría de Desarrollo Social (SEDESOL).

La situación se complicaba con eventos como el asesinato del candidato presidencial Luis Donaldo Colosio, la rebelión del ejército zapatista en Chiapas y el "error de diciembre"4, estos hechos, aunados a la situación financiera por la que atravesaba el país, provocaron otra severa crisis económica. A pesar de lo anterior, nuevamente es elegido un presidente del Partido Revolucionario Institucional (PRI), Ernesto Zedillo Ponce de León, quien enfoca los esfuerzos en la atención de la lucha contra la pobreza, con el Programa de Educación, Salud y Alimentación o PROGRESA, con una cobertura de 2.6 familias en extrema pobreza, cifra que se encontraba muy por debajo del programa PRONASOL, que atendía a la mitad de la población. PROGRESA se dirigió a la pobreza extrema rural, eliminando el subsidio a la tortilla y la leche (CONASUPO), que se consideraban apoyos a la pobreza urbana. PROGRESA apoyó a través de subsidios en efectivo para la alimentación, atención a la salud y becas escolares. De manera paralela surgió el programa PROCAMPO, diseñado para otorgar subsidios a los campesinos para la producción.

En las elecciones del 2000, triunfa la derecha en México, representada por el Partido Acción Nacional con el candidato Vicente Fox Quezada, considerándose que existía una economía estable, pero sin crecimiento. La pobreza y sobre todo la pobreza extrema rural y el desempleo habían aumentado. PROGRESA no había tenido los resultados esperados, por lo que surgió una nueva etapa del programa OPORTUNIDADES que, aunque no estaba enfocado al desarrollo, si pretendía mejorar el nivel de vida de los grupos vulnerables.

Por lo tanto, se puede decir que los programas sociales aplicados desde principios de los años ochenta, resumidos en la tabla "Programas de apoyo rural" (tabla 1), no pudieron lograr la superación de los rezagos de los grupos marginados del país.

${ }^{4}$ Se llamó así al pánico financiero generalizado que provocó el anuncio de la Secretaría de Hacienda de un cambio en la banda de flotación del peso 
Tabla 1

Programas de apoyo rural (2012-2018)

Programas de apoyo rural (2012-2018)

Programa o Institución

Objetivo

Población destinataria

SEDESOL (SECRETARÍA DE DESARROLLO SOCIAL)

Opciones productivas

Atención a Jornaleros Agrícolas

Programa para el Desarrollo de Zonas

Prioritarias (PDZP)

Programa $3 \times 1$ para Migrantes

Fondo para el Apoyo a Proyectos

Productivos en Núcleos Agrarios

(FAPPA)

El Programa de la Mujer en el

Sector

Agrario (Promusag)

Programa Joven Emprendedor

Rural y

Fondo de Tierras

Vivienda Digna

Vivienda Rural

PASPRAH

Apoyo para Regularizar

Asentamientos

Humanos Irregulares (PASPRAH).
Apoya proyectos productivos, sustentables económica y

ambientalmente, mediante la incorporación y desarrollo de capacidades

productivas y técnicas.

Generar igualdad de oportunidades y ampliación de capacidades.

Atiende integralmente los rezagos vinculados con la infraestructura

básica comunitaria, y la carencia de servicios básicos en las viviendas,

ubicadas en los municipios de muy alta y alta marginación que

conforman las ZAP (Zonas de Atención Prioritarias).

Apoya las iniciativas de los migrantes y les brinda la oportunidad de $\quad$ Mexicanos que viven en el exterior.

canalizar recursos a México, en obras de impacto social que benefician

directamente a sus comunidades de origen.

\section{SEDATU (SECRETARÍA DE DESARROLLO AGRARIO, TERRITORIAL Y URBANO)}

Poner en marcha proyectos productivos para mejorar la calidad de vida de los individuos y la de sus familias.

Mujeres y hombres que habitan en núcleos agrarios y que no son titulares de tierras.

\section{Apoyar el desarrollo de un proyecto productivo que permita obtener} ingresos y con ello contribuyan a combatir la pobreza en el medio rural.

Fomentar el relevo generacional en la tenencia de la tierra, apoyando a $\quad$ Jóvenes de núcleos agrarios interesados/as en adquirir 0 los beneficiarios, quienes reciben asistencia técnica y capacitación para rentar tierra social.

instrumentar proyectos productivos sustentables que mejoren sus ingresos y nivel de vida, arraigándolos en sus comunidades.

Se otorgan subsidios para la adquisición, construcción, ampliación 0 mejoramiento de la vivienda.

Hogares mexicanos en situación de pobreza con ingresos por debajo de la línea de bienestar, con carencia de calidad y espacios de la vivienda.

Se otorgan subsidios para la adquisición, construcción, ampliación 0 mejoramiento de la vivienda.

Hogares mexicanos rurales en situación de pobreza con ingresos por debajo de la línea de bienestar, con carencia de calidad y espacios de la vivienda.

Apoya con 10 mil pesos a los hogares en situación de pobreza que habitan en asentamientos humanos irregulares para que cuenten con certeza jurídica respecto a su patrimonio mediante la obtención de su escritura o título de propiedad.

\begin{tabular}{|c|c|c|}
\hline \multicolumn{3}{|c|}{ SAGARPA (SECRETARÍA DE AGRICULTURA, GANADERÍA, DESARROLLO RURAL, PESCA Y ALIMENTACIÓN } \\
\hline PROCAMPO & Apoyar a los productores, ya sean personas físicas o morales. & $\begin{array}{l}\text { Personas físicas o morales que formen parte del "Padrón } \\
\text { de Beneficiarios". }\end{array}$ \\
\hline $\begin{array}{l}\text { DESARROLLO DE CAPACIDADES Y } \\
\text { EXTENSIONISMO RURAL }\end{array}$ & $\begin{array}{l}\text { Fomentar el desarrollo de capacidades de los productores, sus } \\
\text { organizaciones, las familias rurales y otros actores que realizan oficios } \\
\text { en el medio rural, }\end{array}$ & $\begin{array}{l}\text { Personas físicas, morales, grupos con actividades } \\
\text { productivas en el medio rural, e instituciones } \\
\text { especializadas en la capacitación e investigación }\end{array}$ \\
\hline $\begin{array}{l}\text { Fondo para Acciones de } \\
\text { Alimentación en } \\
\text { Concurrencia en Zonas de Alta y } \\
\text { Muy } \\
\text { Alta Marginación } 2012\end{array}$ & $\begin{array}{l}\text { Contribuir a integrar las zonas de alta y muy alta marginación a la } \\
\text { dinámica del desarrollo económico y social, satisfaciendo las } \\
\text { necesidades básicas de alimento, ofreciendo y fortaleciendo alternativas } \\
\text { para la generación de alimentos para el autoconsumo y la } \\
\text { comercialización. }\end{array}$ & $\begin{array}{l}\text { Personas físicas, grupos de trabajo para un propósito } \\
\text { común o personas morales que se ubiquen en } \\
\text { localidades rurales de alta y muy alta marginación, que } \\
\text { se dediquen a actividades agrícolas, pecuarias, } \\
\text { pesqueras, acuícolas y/o, agroindustriales. }\end{array}$ \\
\hline $\begin{array}{l}\text { Proyecto Estratégico de Seguridad } \\
\text { Alimentaria (PESA) }\end{array}$ & $\begin{array}{l}\text { Contribuir al desarrollo de capacidades de las personas y su agricultura y } \\
\text { ganadería familiar en localidades rurales de alta y muy alta marginación, } \\
\text { para incrementar la producción agropecuaria, innovar los sistemas de } \\
\text { producción, desarrollar los mercados locales, promover el uso de } \\
\text { alimentos y la generación de empleos a fin de lograr su seguridad } \\
\text { alimentaria y el incremento en el ingreso. }\end{array}$ & $\begin{array}{l}\text { Personas físicas, grupos de trabajo para un propósito } \\
\text { común o personas morales, que se ubiquen en } \\
\text { localidades rurales de alta y muy alta marginación de las } \\
\text { Entidades Federativas con mayor grado de marginación y } \\
\text { pobreza del país. }\end{array}$ \\
\hline $\begin{array}{l}\text { Desarrollo de las Zonas Áridas } \\
\text { (PRODEZA) }\end{array}$ & $\begin{array}{l}\text { Impulsar y fomentar la inversión social y privada en las zonas del trópico } \\
\text { húmedo y sub-húmedo del territorio nacional, a través del otorgamiento } \\
\text { de apoyos para mejorar la viabilidad financiera de proyectos de cultivos y } \\
\text { actividades con potencial y mercado, }\end{array}$ & $\begin{array}{l}\text { a) Beneficiarios con crédito y/o con financiamiento propio. } \\
\text { b) Beneficiarios de apoyo directo. }\end{array}$ \\
\hline
\end{tabular}

Fuente : Elaboración propia con base en diversas fuentes 
En el período comprendido entre 1980 y 1989, Banrural habilitó crediticiamente, en promedio, 6 millones 590 mil hectáreas. En la siguiente década la superficie acreditada fue de 1 millón 608 mil, registrando una disminución de $76 \%$. Otras problemáticas importantes que señalaban la urgente necesidad de un cambio en el sistema financiero mexicano, era la incapacidad de muchos productores para ser considerados sujetos de crédito, la deficiente asignación de recursos para la inversión y la dependencia permanente de los subsidios; por lo que, fue necesario realizar una reforma radical, que inició en 1988 y que se caracterizó por:

- Se eliminó el “encaje legal”, en 1988

- Se reprivatizó la Banca Comercial, en 1991 (nacionalizada en 1982)

- Se realizaron reformas legales importantes, en la Ley para Instituciones de Crédito y Mercado de Valores y la Constitución para establecer un régimen mixto de banca y crédito, autorizado en 1990.

- Se reunieron las micro financieras para consolidarse como Entidades de Ahorro y Crédito Popular (EACP) y cumplir con lo establecido en la Ley de Ahorro y Crédito Popular (LACP)

- Se instauraron tasas de interés variable, para que los réditos que se cobraran en el sector agrícola pudieran ser tasas positivas en términos reales.

- Se desapareció Aseguradora Nacional de la Agricultura y Ganadería (ANAGSA) y se crea Agroaseguradora Mexicana (AGROASEMEX), con lo que la recuperación de los créditos a través de los seguros se elimina

- Se establecieron objetivos de rentabilidad de Banrural, dándole la libertad de financiar cualquier actividad (de 1988 a 1994 las sucursales se redujeron de 650 a 230 y el número de empleados de 27,000 a 9,000)

- Se liquidó el fondo de fomento que atendía a Banrural, dejándole a Nafin, Bancomext y Fideicomisos Instituidos en Relación con la Agricultura de la Banca de México (FIRA) como fuentes de fondeo, con lo que igualó sus condiciones con la Banca Comercial.

- Se impusieron garantías adicionales a los créditos otorgados por Banrural, eliminando las reestructuras generalizadas y obligando a realizar un análisis de riesgo para cada solicitud de financiamiento

- Se dividieron las funciones de las entidades que conformaban el Sistema Financiero Rural, de la siguiente manera:

- PRONASOL atendería a los productores ubicados en zonas de siniestralidad recurrente y baja productividad, en donde recibirían créditos a la palabra con montos bajos y a tasa cero

- Banrural, con apoyo de FIRA, se ocuparía de los campesinos de bajos ingresos, pero con potencial productivo.

- Banca comercial en coordinación con los FIRA, Nafin y Bancomext, atendería a los medianos y grandes productores.

Después de la reforma financiera, en la década de los noventa, se consideró la posibilidad de que las fuentes de financiamiento privadas tuvieran la intención de invertir en el campo, con base en que su participación aumentó de manera importante. Sin embargo, la realidad de esta percepción era que FIRA otorgó una gran cantidad de recursos financieros en estímulos y garantías representadas en porcentajes de intermediación, estímulos compensatorios especiales y garantías para la inversión, de manera que propició que la Banca Comercial pudiera participar de manera segura, hasta que la crisis registrada en 1995, en donde la participación privada disminuyó significativamente.

De acuerdo al INEGI, en 2004, habiéndose establecido la nueva estructura de Financiera Rural, se registra un incremento en la colocación de créditos de 52\% en 2005. Para el siguiente año disminuye y solamente crece un

\footnotetext{
${ }^{5}$ De acuerdo al Banco de México, el encaje legal era la fracción obligatoria mínima (requerida por la autoridad, a través del Banco Central) del depósito de los ahorradores, que los bancos comerciales deben mantener como reservas para poder atender los retiros de los depositantes
} 
9.3\%, para $200736.1 \%$, para $200832.2 \%$ y para el 2009 sufre un descenso significativo, registrando un 8.9\%, debido a los efectos de la crisis de Estados Unidos.

Las características observadas en los créditos agrícolas colocados en ese período fueron que la zona norte fue en donde se colocaron la mayor cantidad de créditos (79\%) y por estados eran Sinaloa $13 \%$, Jalisco $9 \%$, Sonora $8 \%$, Chihuahua ${ }^{6} 8 \%$, Veracruz $7 \%$ y Guanajuato $6 \%$. De acuerdo al destino que se les dio el más demandado fue el simple en 45\%, el de habilitación o avío en 32\%, prendario 16\% y refaccionario 5\%. (Eligio \& Gómez Gómez, 2013)

En resumen, en las regiones norte y noroeste, conformados por 9 estados, se colocaron el $46 \%$ de los recursos, mientras que, en el sur y sureste, integradas por 13 estados, se concentró el $25 \%$ del monto total colocado por FIRA.

Con respecto a FIRA, entre $2009-2010$ el $40 \%$ de su colocación fue para productores con ingresos anuales menores a 3 mil veces el salario mínimo, el resto para los que registraron un ingreso mayor. (Banxico, 2010). No obstante, entre 2000 y 2005 los fondos FIRA dejaron de incorporar a pequeños y medianos productores, registrando un crecimiento de $92 \%$ aproximadamente, por lo que se puede deducir que, en ese periodo el crédito estaba concentrado con las empresas medianas y grandes. Otra situación que se observó del 2004 al 2009, fue la disminución del otorgamiento de créditos refaccionarios, que sufrieron un decremento promedio anual del $10 \%$.

Con respecto a la recuperación de los recursos otorgados, los niveles de cartera vencida observados eran bajos, demostrando que la mayor parte de los proyectos apoyados eran viables.

En lo que respecta a la Banca Comercial, la colocación de créditos registró un crecimiento promedio anual del 4\%. De 2004 a 2005 disminuyó un 19\%, en los años subsecuentes, es decir 2006, 2007 y 2008 aumenta un 1\%,30\% y $27 \%$, para el 2009 nuevamente disminuye un 10\%. Con respecto a las zonas atendidas, la más beneficiada es la Ciudad de México (CDMX) con una colocación promedio del 12.5\% del total, seguida por el Estado de Sonora con un $11.5 \%$, Sinaloa el $10.8 \%$, Coahuila el $7.6 \%$, Jalisco el $6.9 \%$, Guanajuato el $5.9 \%$, Durango con el $4.5 \%$ y Chihuahua el 4.1\%. (Eligio \& Gómez Gómez, 2013).

Considerando lo anterior se puede suponer que el financiamiento rural podría mejorar, sin embargo, se observaban varios aspectos preocupantes, el primero estaba representado por la falta de atención de los pequeños y medianos productores del sur y sureste del país, en donde se encontraban los menores niveles de capitalización y productividad; el fenómeno era ocasionado por FIRA, Financiera Rural y por la Banca Comercial, perpetuando el dualismo estructural que ha imperado desde hace varias décadas. El segundo aspecto era la disminución del crédito refaccionario, en donde la oferta financiera de Financiera rural fue mayor que la de FIRA.

\subsection{El Sistema Producto Arroz en Morelos}

El objeto de estudio es el Sistema Producto Arroz de Morelos. Su representante y presidente de la Asociación de Arroceros de la Región Sur del Estado de Morelos USPR, Don Jesús Solís Alvarado, cuida los intereses de 13 sociedades de producción rural con 250 productores que, en total, incluyendo los jornaleros que contratan, significan 600 puestos de trabajo agrícola en el Estado. La producción de la asociación se procesa y envasa en el Molino de arroz San José, ubicado en el municipio de Jojutla de Juárez en Morelos. Se trata de uno de los molinos más antiguos que existen en Morelos. (Solís Alvarado, 2018). El arroz fue uno de los granos básicos más afectados

\footnotetext{
${ }^{6}$ El estado de Chihuahua registró un crecimiento promedio anual de $42 \%$, participando con un $8 \%$ del total de los créditos colocados, “Análisis de la política de financiamiento rural en México" ,2013
} 
por la implantación de la política neoliberal de Carlos Salinas de Gortari, la desaparición de los precios de garantía (1989) y la falta protección frente a los mercados internacionales que eran subvencionados y subsidiados, fueron los factores de mayor incidencia en la quiebra del Molino de arroz San José. Aunado a lo anterior, de acuerdo a Steffen Riedeman, el gobernador electo Antonio Rivapalacio, respaldo el endeudamiento del Molino y permitió que los recursos se utilizaran para otros fines (Ramírez, Valdés , \& Vivar, 2015). Lo hizo a través de subordinar a los arroceros a un empresario privado, Ángel Morales Orañegui, (1992) cuya familia estaba vinculada con las personas que representaban el poder político.

Dos años más tarde, como resultado de la deficiente y corrupta administración, el gobernador le regresó el Molino a la Asociación de Arroceros del estado de Morelos, sin embargo, ellos desconocían era que existía una deuda de 20 millones de pesos, que desde esa fecha fueron responsables de pagar. El apalancamiento heredado, además de impedirles solicitar recursos por no ser sujetos de crédito, los obligó a enfrentar un gran número de demandas, hasta el 2005, cuando se publicó en el Diario Oficial de la Federación que la deuda sería condonada y el Molino donado a la Asociación afectada.

Sin embargo, fue hasta noviembre del 2016, cuando la diputada Hortencia Figueroa Peralta, exhortó al Congreso del Estado para cumplir el decreto con fecha 5 de febrero del 2005, en el que se disponía tal medida. La relación de los productores con el Molino siempre ha sido motivo de disputa, se cree que también provocada por las entidades de gobierno para poder controlar el medio rural. La infuencia de las entidades gubernamentales también se observa en la comercialización del grano, el precio es determinado por los 4 molinos que operan en el Estado y también por SAGARPA y SEDAGRO, sin la participación de los productores base. Por otro lado, el pago del arroz a los productores por parte de los molinos puede tardar hasta 1 año.

\subsection{Estudio de campo}

Se desarrolló un instrumento compuesto de 3 secciones con un total de 60 preguntas que comprendía aspectos estratégicos del alcance, amplitud y profundidad de los programas de apoyo.

\section{Gráfica 1}

Destino de los recursos financieros que recibieron los productores de arroz en Morelos. Fuente:

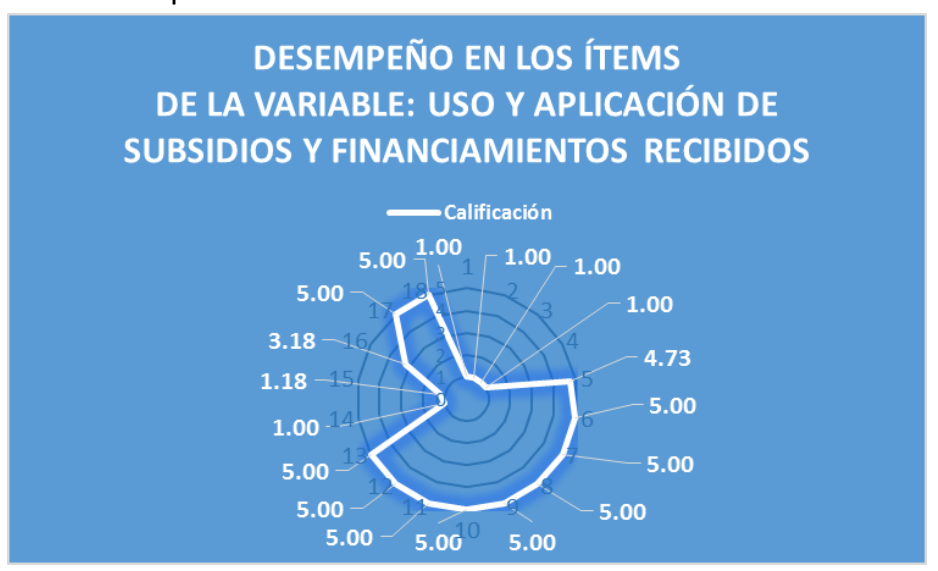

Elaboración propia

De acuerdo a los productores, los principales destinos de los recursos solicitados por los productores son: 1. Resiembras, 2. Compra de gasolina, diésel y aceites aditivos, 3. Renta de maquinaria, equipo e instalaciones, 4. Arrendamiento de tierras, 5. Mejoramiento genético, 6. Servicios de asistencia técnica, 7. Consumo de energía eléctrica, 8. Fletes y transportes, 9. Sistemas de información y 9. Derecho de agua para riego. 
En lo que respecta al uso, el orden de importancia se aplica para: 1. Compra de semillas o plantas, 2. Adquisiciones de abonos, fertilizantes, insecticidas y plaguicidas, 3. Preparación del suelo en las labores agrícolas, 4. Labores de cosecha, 5. Impuestos o intereses y por último 6. Semillas mejoradas.

Los resultados mencionados nos indican que no existe un patrón en el destino de los recursos que obtienen los arroceros de Morelos, es decir, no existe planeación en las ofertas de apoyo y planes específicos en cada programa.

\section{Gráfica 2}

Aplicación de los subsidios

y financiamientos

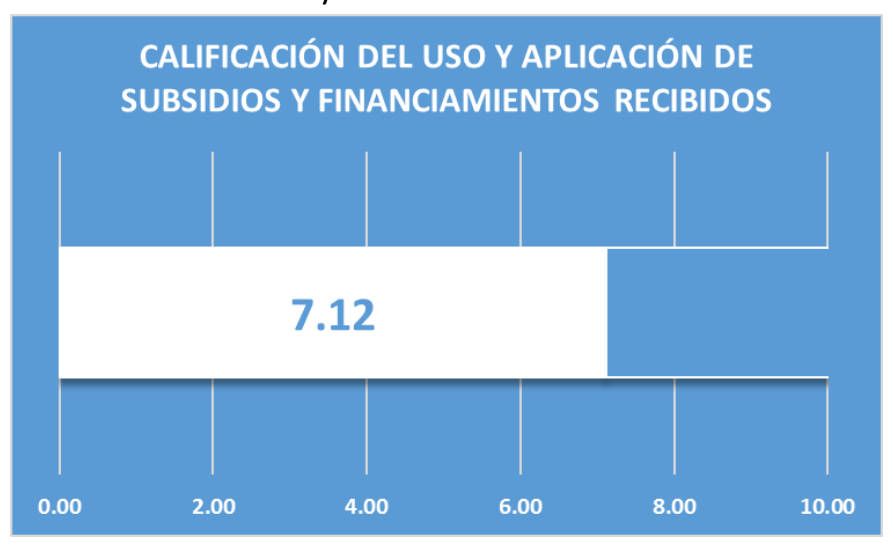

Fuente: Elaboración propia

Se analizó la información acerca del destino de los recursos provenientes de los financiamientos que les otorgan, buscando un criterio de clasificación, resultado de un patrón, es decir, poder concluir que se otorgan a un propósito general como la compra de maquinaria o la renta de la tierra. Sin embargo, al no observarse dicha situación, se puede deducir que no existe planeación del destino de los recursos y que los agricultores los usan de acuerdo a las necesidades inmediatas que sea necesario cubrir en el momento que lo reciben, porque no son considerados los destinos y a oportunidad en la que se requieren los apoyos

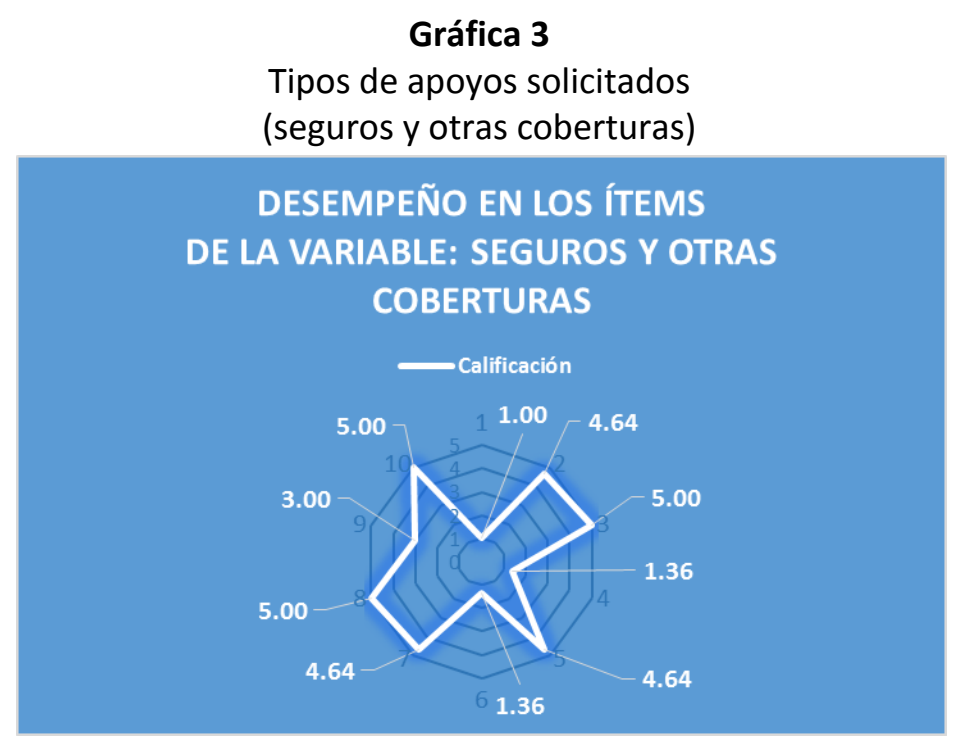

Fuente: Elaboración propia 
Los resultados obtenidos nos demuestran que no se fomenta la cultura de previsión, debido a que los representantes no consideraron necesario contratar un seguro u otro tipo de cobertura. Sin embargo, en los pocos casos que lo han hecho, la mayoría de ellos han acudido a un Fondo de Aseguramiento, algunos con una Institución de Gobierno y en escasas ocasiones con Aseguradoras Privadas.

Por otro lado, se pudo comprobar que conocen el término "garantías líquidas" y, aunque no las utilizan con regularidad, si las han usado en algunas ocasiones.

\section{Gráfica 4}

Conocimiento sobre los apoyos

financieros gubernamentales

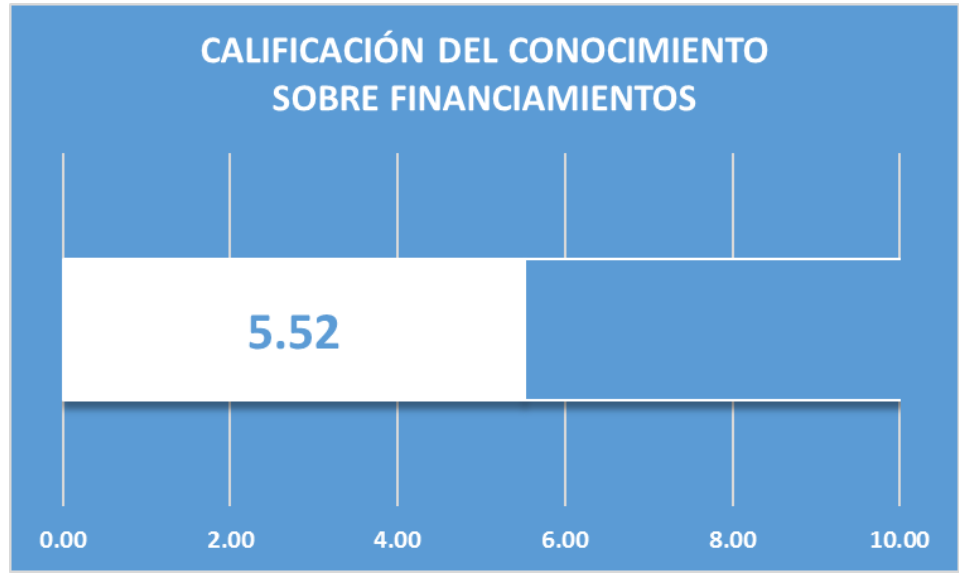

Fuente: Elaboración propia

En general, el conocimiento de los productores de arroz de los programas de apoyo financiero ofrecidos y los trámites que se necesitan para acceder a ellos no superan el $60 \%$.

\section{Gráfica 5}

Fuentes de financiamiento

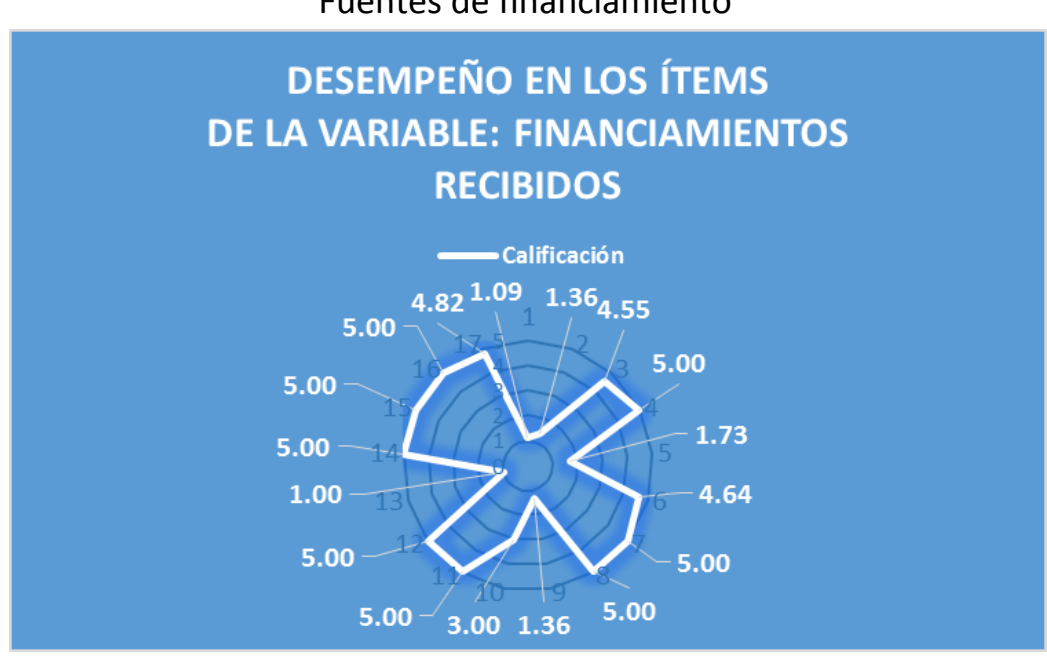

Fuente: Elaboración propia 
Los créditos que reciben los arroceros de Jojutla provienen indistintamente de diversas fuentes como son, en primer lugar: prestamistas, familiares que residen en el extranjero, Uniones de Crédito y por familiares que viven en el país. Después de ellos, las fuentes de financiamientos a las que recurren son SOFOMES, Financiera Rural y por último FIRA

Con respecto a las tasas de interés, la que pagan con mayor frecuencia oscila en el rango de 25 a 40 puntos y en algunos casos mayor del 40\%. También reportaron que, en algunas ocasiones, han podido pagar entre el 15 y el $25 \%$ (pocos casos). Existen casos aislados donde pagaron menos del $15 \%$.

Los rangos de plazos que les fueron otorgados con mayor frecuencia son: 1. De tres a diez años, 2. De diez a veinte años y 3. De veinte años en adelante. El tiempo de pago les fue autorizado en función del tipo de financiamiento solicitado, el crédito para capital de trabajo (líneas de crédito) y para insumos (habilitación o avío) no podrá exceder de 10 años y los que destinen a la compra de maquinaria y equipo o sean para la construcción de naves industriales (refaccionarios) podrán exceder ese tiempo ampliamente, de acuerdo a su naturaleza y tiempo de ejecución.

Por último, confirmaron que la oferta de productos financieros por parte de las instituciones antes mencionada es consistente lo que le da sostenibilidad a la oferta crediticia.

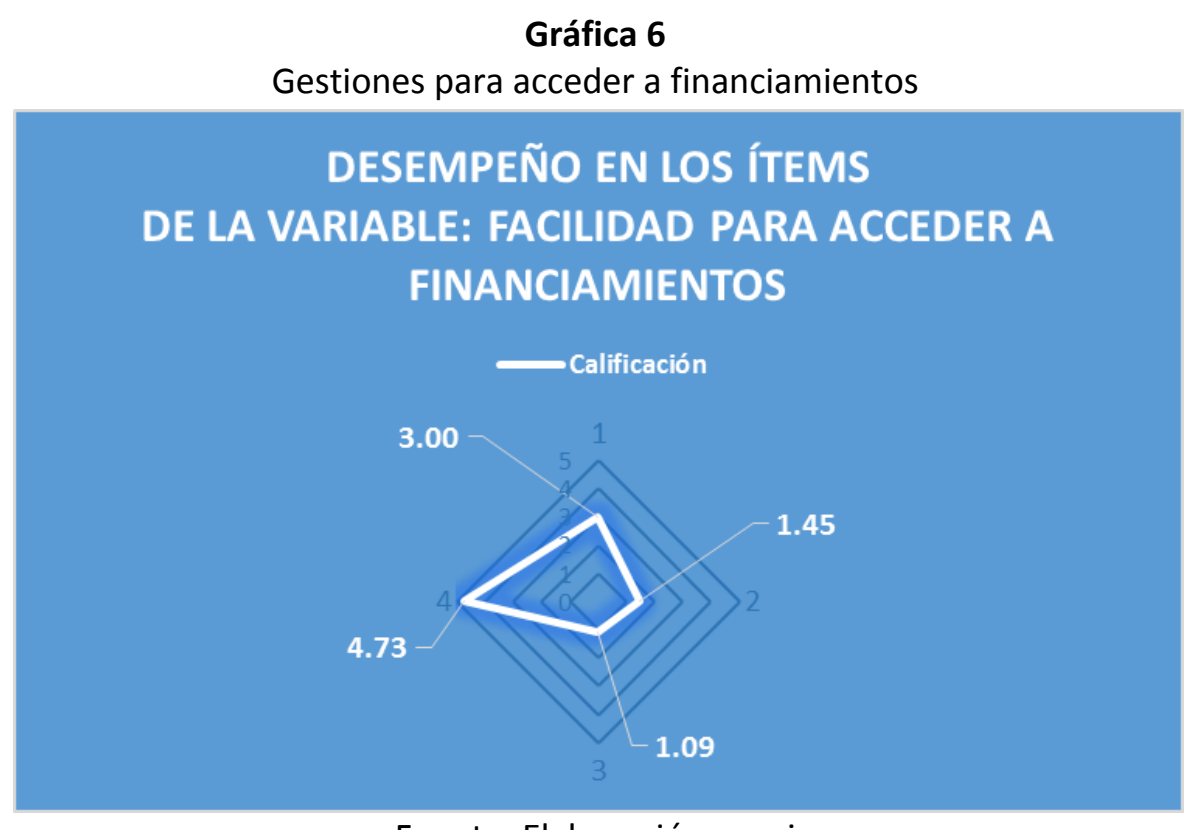

. Fuente: Elaboración propia

La complejidad de las gestiones necesarias para obtener un financiamiento fue calificada como difícil, superior al promedio. Existen diversos motivos para que les rechacen las solicitudes de crédito. Se partió de la suposición de que no les eran otorgados los financiamientos por carecer de las características necesarias para ser sujetos de crédito o por no tener el respaldo económico requerido; sin embargo, dichas situaciones no fueron los motivos por los que les niegan los recursos. Las razones por las que no son considerados por los programas de apoyo, son la ausencia de liquidez y capacidad de pago. La primera derivada de la estacionalidad de la venta de su producción y la segunda de los bajos márgenes de rentabilidad con los que operan. 
Gráfica 7

Facilidad para acceder a los financiamientos

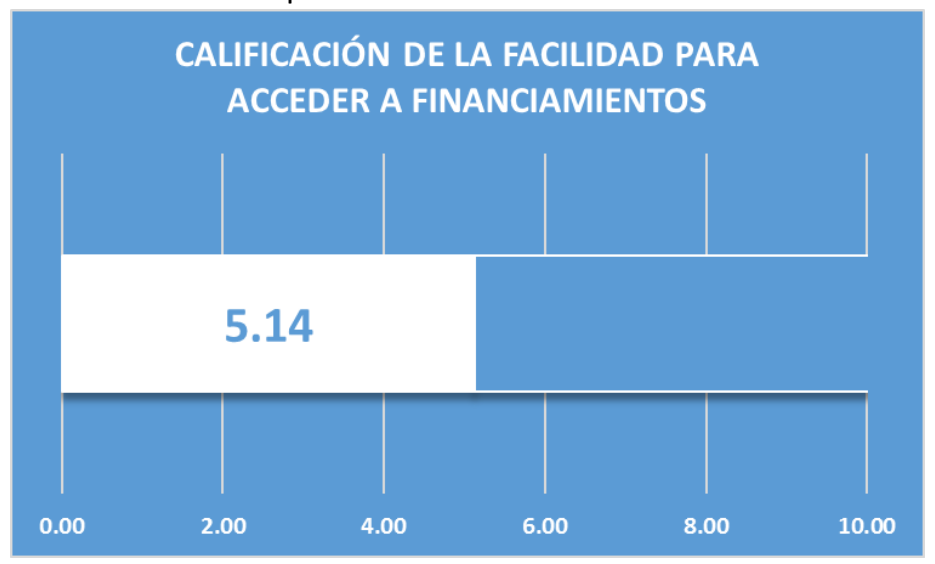

Fuente: elaboración propia

La percepción de los arroceros en relación a la facilidad de obtener un crédito es ligeramente superior al promedio, descartando a la ausencia de propiedades que puedan ser usadas como garantía y el riesgo del tipo de actividad que realizan.

Cabe mencionar que el cultivo del arroz es una actividad económica de alto riesgo, debido a que su producción depende de factores exógenos, como sequías o plagas, entre otros. Otro de los aspectos que influye de manera importante en aumentar el riesgo son los precios en los que pueden colocar el producto en el mercado, los cuales dependen de los estándares internacionales determinados en Bolsas de Valores como la de Chicago (Chicago Mercantile Exchange) o la de Nueva York (New York Mercantile Exchange).

\section{Conclusiones}

La investigación realizada permitió determinar que las características de los programas de apoyo gubernamentales, con respecto a la diversidad de los servicios financieros ofrecidos (amplitud), es insuficiente, principalmente porque no consideraron el destino que le dieron los agricultores a los recursos. Por otro lado, tampoco se observa que las solicitudes de ayuda de los campesinos de Jojutla de Juárez, hayan podido ser atendidas, confirmando la ausencia de alcance, debido a que su principal fuente de financiamiento fueron prestamistas y familiares que residían en el extranjero. Por último, quedó en evidencia que el $60 \%$ de los productores no conoce los programas y, por lo tanto, tampoco los trámites necesarios para acceder a ellos, lo que confirma la falta de profundidad de la ayuda.

\section{Referencias bibliográficas}

Banxico. (2010). Informe anual. CDMX: Banco de México.

CEFP. (2007). Los subsidios agrícolas en los países del TLCAN. CDMX: Gobierno de la República.

Cordera, R., \& Lomelí, L. (2005). La política social moderna: evolución y perspectivas; resumen ejecutivo y consideraciones finales. Cuadernos de Desarrollo Humano.

Cruz, M., \& Polanco, M. (2014). El sector primario y el estancamiento económico en México. Problemas del Desarrollo, 9-33.

De la Vega Mena, M., Santoyo Cortes, V. H., Muñóz Rodríguez, M., \& Altamirano Cárdena, J. (2014). Cobertura financiera de la Banca de desarrollo para el sector rural de México: FIRA y Financiera Rural. Estudios Sociales, 225-248. 
Delgado, L. (1991). Production alimentaire et reproduction sociale, I alimentation dans le processus de transation des communautés payssanes péruviennes. Thése de doctorat nouveau régime, Université de Paris I-Pnatheon-Sorbonne. Paris, Francia, IEDES.

DOF. (diciembre de 2001). Ley de Desarrollo Rural Sustentable. Diario Oficial de la Federación, pág. 57.

Eligio, F. G., \& Gómez Gómez, A. A. (2013). Análisis de la política de financiamiento rural en México 1990-2010. Revista mexicana de Ciencias Agrícolas, 1109-1117.

FAO. (13 de febrero de 2013). Índice de la FAO para los precios de los alimentos. Obtenido de Food and Agriculture Organization: http://www.fao.org/worldfoodsituation/wfs-home/foodpricesindex/es/

Galindo Olguín, M. A., \& Sainz Acosta, J. G. (2014). EL agro mexicano en el siglo XXI. En G. Torres Salcido , \& M. Morales Ibarra, El agro y las áreas rurales en el México del siglo XXI (págs. 19-34). México: UNAM.

GEA . (2011). Desventajas y riesgos del libre comercio agropecuario con Brasil, Colombia y Perú. México: Grupo de Economistas y Asociados.

GEA. (2012). Propuesta de modelo. Política pública para el sector agroalimentario mexicano. Importancia del sector en México, México. México: Grupo de Economistas y Asociados.

GRUPO INTERAGENCIAL DEL DESARROLLO RURAL, IICA,BID,CEPAL,FIDA,GTZ,BANCO MUNDIAL,USAID. (2014). Más que alimentos en la mesa: la real contribución de la agricultura en la economía. IICA.

Herrera, F. (2009). Apuntes sobre las instituciones y los programas de desarrollo rural en México. Del Estado benefactor al Estado neoliberal. Centro de investigación en alimentación y desarrollo, 1-34.

Medina Macias, A., \& Ávila Vidal, A. (2002). Evolución de la teoría administrativa. Una visión de la psicología organizacional. Revista cubana de psicología, 262-272.

Miller, E. (1985). Desarrollo integral del medio rural. Un experimento en México. Fondo de cultura económica.

Ramírez, l., Valdés , L., \& Vivar, R. (21 de 08 de 2015). Organización y sobrevivencia de los campesinos arroceros de Morelos. (M. Steffen, Entrevistador)

Reig, N. (2004). América Latina y la mundialización agropecuaria. En M. d. Del Valle Rivera, El desarrollo agrícola y rural del tercer mundo en el contexto de la mundialización (págs. 23-54). CDMX: UNAM.

Rodriguez, D. R. (2005). El caso de la agroindustria panadera en Colombia. Perspectivas rurales. En R. G. D., La multifuncionalidad de los SIALES en zonas rurales de paises en desarollo. (págs. 133-125). Colombia: Universidad de Colombia.

Rosales, R. (2010). Aprendizaje colectivo, redes sociales e instituciones. Hacia una nueva geografía económica. Los giros de la geografía humana: desafíos y horizontes, 123-142.

SAGARPA/FAO. (2016). Implementación de estategias y proyectos para la pequeña agricultura en zonas rurales marginadas. Ciudad de México: FAO.

Salinas Callejas, E. (1995). El financiamiento del sector agropecuario en México, 1988-1994. Revistas Bancomext.

Sánchez Bañuelos, M. N. (2017). Aportes teóricos a la gestión organizacional: La evolución en la visión de la organización. Revista digital/FCE/UNLP, 65-75. 
Sanz Cañada, J. (2014). Sistemas Agroalimentarios Locales y multifuncionalidad. Un enfoque de investigación en alimentos, ciencias sociales y territorio. En M. Del Valle Rivera, El desarrollo hoy. Hacia la construcción de nuevos paradigmas (págs. 87-104). Ciudad de México: Insituto de Investigaciones Económicas.

Solís Alvarado, J. (4 de octubre de 2018). Eficacia de apoyos financieros. (A. Fernández Hernández, Entrevistador)

Steffen, C. (2015). Los campesinos productores de arroz del estado de Morelos: un futuro incierto. Artículos y Ensayos de la Sociología Rural, número 20, 69-89.

Torre, A., \& Filippi, M. (2005). Proximitès et chengements socio-économiques dans les mondes ruraux. París, inra, 322.

Torres Salcido, G., Sanz Cañada, J., \& Muchnik. (2010). Territorios Rurales, Pobreza, accion colectiva y multifuncionalidad, México. UNAM, 388.

Zorrilla Ornelas, L. (2003). Las políticas mexicanas de desarrollo rural en el siglo xx. Comercio Exterior, 104-113.

Esta obra está bajo una Licencia Creative Commons Attribución-NoCommercial 4.0 International

(cc) EY-NC 